LA-UR-01-4539

Approved for public release; distribution is unlimited.

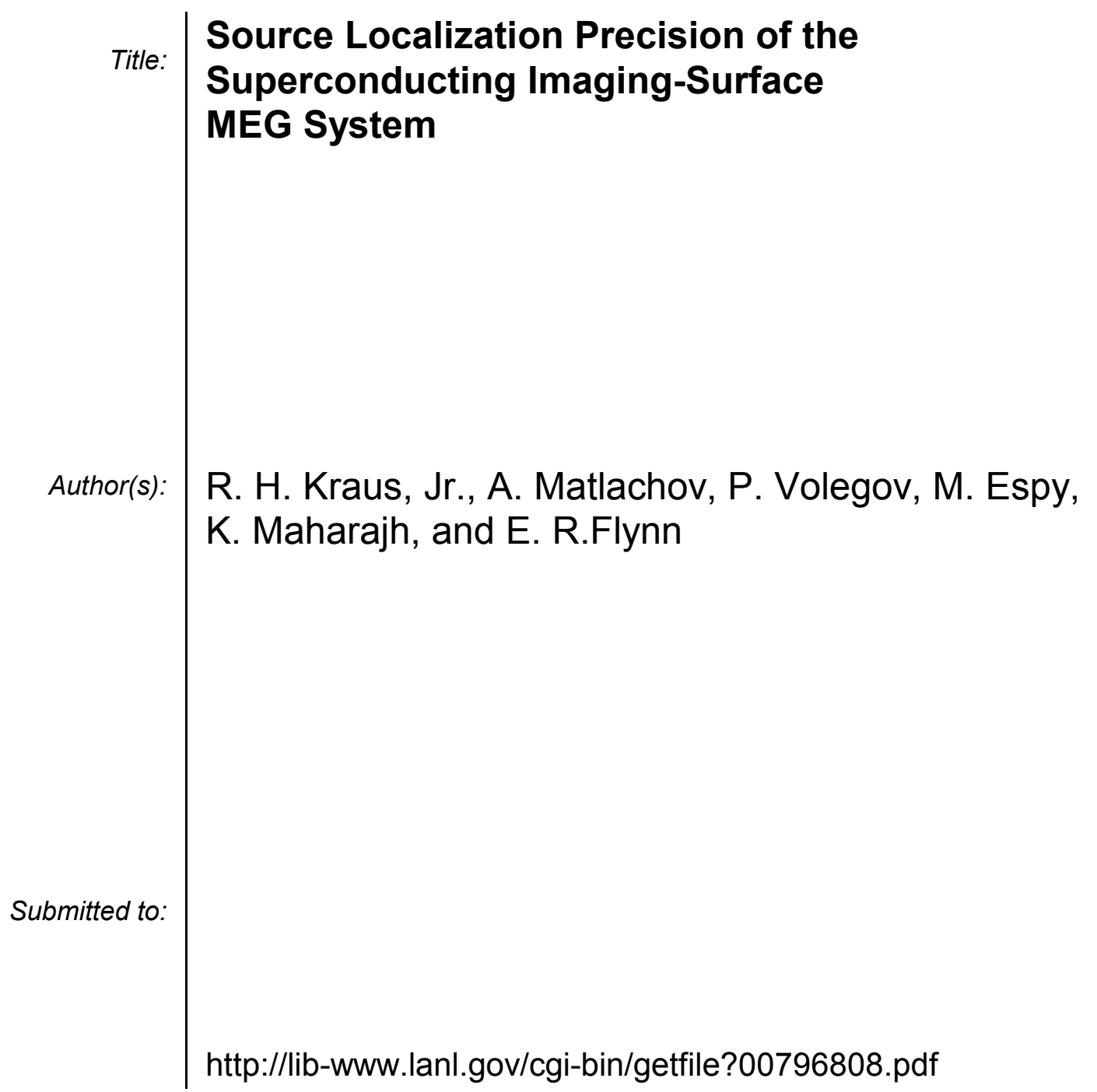

Los Alamos National Laboratory, an affirmative action/equal opportunity employer, is operated by the University of California for the U.S. Department of Energy under contract W-7405-ENG-36. By acceptance of this article, the publisher recognizes that the U.S. Government retains a nonexclusive, royaltyfree license to publish or reproduce the published form of this contribution, or to allow others to do so, for U.S. Government purposes. Los Alamos National Laboratory requests that the publisher identify this article as work performed under the auspices of the U.S. Department of Energy. Los Alamos National Laboratory strongly supports academic freedom and a researcher's right to publish; as an institution, however, the Laboratory does not endorse the viewpoint of a publication or guarantee its technical correctness. 


\title{
Source Localization Precision of the Superconducting Imaging-Surface MEG System
}

\author{
Kraus, Jr., R.H., Matlachov, A., Volegov, P., Espy, M., Maharajh, K., and E.R.Flynn \\ Los Alamos National Laboratory, Los Alamos, NM USA \\ University of New Mexico, Albuquerque, NM, USA
}

\section{Introduction}

A unique whole-head Magnetoencephalography (MEG) system incorporating a superconducting imaging surface (SIS) has been designed and built at Los Alamos with the goal of dramatically improving source localization accuracy while mitigating limitations of current systems (e.g. low signal-to-noise, cost, bulk). Magnetoencephalography (MEG) measures the weak magnetic fields emanating from the brain as a direct consequence of the neuronal currents resulting from brain function[1]. The extraordinarily weak magnetic fields are measured by an array of SQUID (Superconducting QUantum Interference Device) sensors. The position and vector characteristics of these neuronal sources can be estimated from the inverse solution of the field distribution at the surface of the head. In addition, MEG temporal resolution is unsurpassed by any other method currently used for brain imaging. Although MEG source reconstruction is limited by solutions of the electromagnetic inverse problem, constraints used for source localization produce reliable results.

The Los Alamos SIS-MEG system[2] is based on the principal that fields from nearby sources measured by a SQUID sensor array while the SIS shields the sensor aray from distant noise fields. In general, Meissner currents flow in the surface of superconductors, preventing any significant penetration of magnetic fields. A hemispherical SIS with a brim, or helmet, surrounds the SQUID sensor array largely sheilding the SQUIDs from sources outside the helmet while measuring fields from nearby sources within the helmet.

We have implemented a finite element model (FEM) description of the SIS using the exact as-built geometry to accurately describe how the SIS impacts the forward physics of source models. The FEM is used to calculate the distribution of Meissner currents in the complicated surface geometry of the SIS such that $\mathrm{B}_{\perp}=0$ at the surface. This model of the forward physics is described elsewhere in these proceedings [3]. In this paper, we present the results of localizing well characterized phantom sources using the SIS-MEG system, the SIS forward model, and a simple inverse method.

\section{Method}

The SIS is fabricated from lead (Type I superconductor below $\sim 7$ Kelvins) with a complex shape consisting of a 5.064inch radius hemisphere with two small "cut-outs" at opposite sides of the hemisphere, and a 2-inch brim that is smoothly melded to the edge of the modified hemisphere. An array of 150 SQUID magnetometers is mounted on "studs" at offsets from the SIS ranging from $1 \mathrm{~cm}$ to $3 \mathrm{~cm}$. The SQUID-SIS offsets were allowed to vary in order to place each sensor as close to the headshaped dewar surface, and consequently the subject head, as possible. The entire system is operated in a liquid helium bath at $\sim 4$ Kelvins temperature.

Localizing sources of neuronal activation from MEG measurements requires a complete description of the "forward physics" that describes how neuronal currents lead to magnetic fields at the SQUID sensors. The MEG forward model must include the complex neuronal source model that incorporates intracellular ionic currents, intercellular and extracellular volume currents, brain structure, and conductivities. The forward model for our SIS-MEG system must further include the effect of the superconducting surface on the fields generated by the primary sources.

Magnetic fields produced by sources inside the helmet (e.g. from a subject brain or a 'calibration phantom') are detected by the SQUID magnetometers. Various sets of fixed magnetic dipole coils known as a 'phantoms' (that emulate signals produced by the human brain) have been constructed to measure the effects of the SIS in the Los Alamos MEG system and quantify localization and overall system performance. Simple dry-wire phantoms, for which the source model can be completely described, were chosen to eliminate any source model dependence from our results. The phantoms distributed 25 coils throughout the volume encompassed by the array (e.g. the brain volume), including several coils near the periphery of the sensor array. The computed magnetic field distributions for the 25 phantom coil positions used are shown in Figure 1, clearly illustrating the proximity of several coils near the edge of the sensor array. Three orthogonal

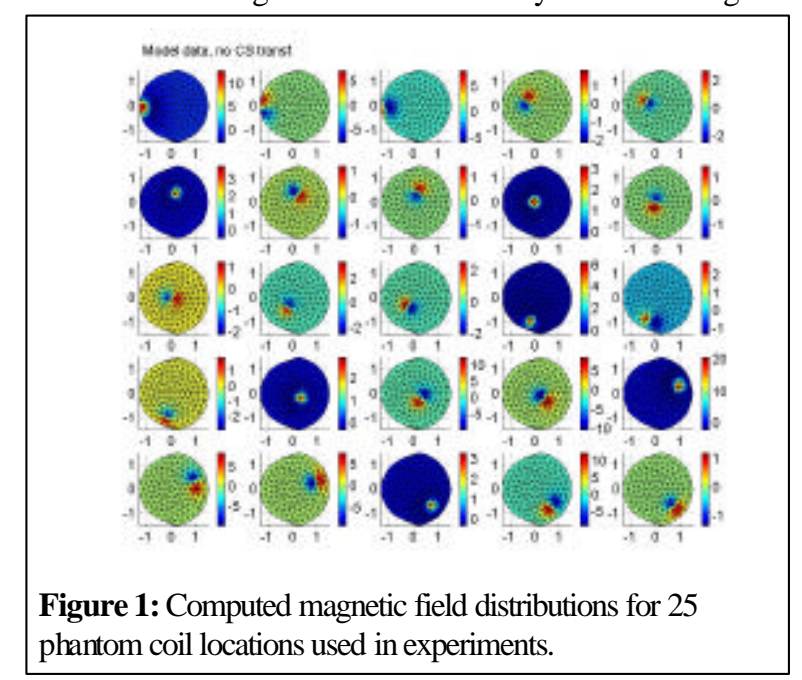


coils were located at most positions to determine if localization accuracy correlated with source orientation. Each phantom coil was precisely machined to a $1.5 \mathrm{~mm}$ radius and located relative to the phantom 'origin' to better than $25 \mu \mathrm{m}$ and $10 \mathrm{mRad}$ accuracy by the Los Alamos mechanical inspection facility.

While the relative position and orientation of each phantom coil is precisely known, the a priori position of the phantom relative to the SQUID array is only known to $\sim \pm 1 \mathrm{~cm}$. Although the SQUID array was fabricated to better than $\pm 50 \mu \mathrm{m}$ overall tolerance, cooling from $\sim 300 \mathrm{~K}$ to $4 \mathrm{~K}$ is expected to result in significant symmetric and asymmetric contractions; consequently we assumed initial $\pm 1 \mathrm{~mm}$ and $\pm 100 \mathrm{mRad}$ sensor position and orientation accuracy. SQUID sensitivities were calibrated prior to installation into the MEG system to a precision of $0.5 \%$, however a systematic error discovered later may increase the total error to as much as $1 \%$.

Phantom source coils were activated using a constant-current supply sinusoidally driven at $77 \mathrm{~Hz}$. The current supply had better than $0.5 \%$ absolute accuracy and $0.1 \%$ stability. A consequence of tolerance build-up in fabricating the phantom coils, it was further necessary to measure the relative field generated by each phantom source coil.

Data were acquired for all 150 SQUID sensors while each phantom coil was excited individually by a currentregulated signal generator at $77 \mathrm{~Hz}$. Data were acquired by simultaneously sampling 24-bit Delta-Sigma digitizers at $3 \mathrm{kSa} / \mathrm{sec}$ at each of the 150 sensor locations. The digitizers were run with high-pass filters disabled and lowpass anti-aliasing filters at $1.2 \mathrm{kHz}$. The raw data were digitally filtered using a band-pass algorithm that produced minimal artifacts was implemented in MATLAB and signal amplitudes for each spectra were determined using standard FFT techniques.

Signals were measured from each phantom coil by all SQUID sensors in the whole-head SIS array for two independent locations of the phantom within the SIS array. Consequently, data for 50 separate phantom locations representing multiple source orientations at most locations were measured. An inversion of the data from one set of 25 phantom locations was performed to localize each sensor in space and orientation. This is conceptually opposite to but mathematically identical to inversion of sensor array data to localize a source. This unique procedure was used to calibrate the position of each sensor at $4 \mathrm{~K}$, compensating for all machining errors and thermal expansions experienced by the system. This provided a correction to the sensor geometry array produced from original system machining diagrams. The resulting "cold" sensor geometry array was used in all subsequent phantom coil localization procedures (using typical inverse methods).

After spatial calibration of the sensor array, independent sets of phantom data were acquired at typical $\mathrm{S} / \mathrm{N}=20$ (100-500fT signal amplitude). Source localization of phantom coils was performed using a simple SVD optimizer (MATLAB) on our source forward physics description. All phantom sources were excited and localized as single sources.

\section{Results}

Efficacy of the basic physics described by the FEM was demonstrated by the excellent agreement between model-calculated and measured magnetic field distribution at the sensors for all phantom coil positions and onientations[3].

Shielding of the SQUIDs from sources external to the array (noise) was both modeled and measured for point dipolar and uniform fields. A measure of how well the SQUID sensors are shielded from external source fields is the 'shielding factor' that we define as the ratio of the free-space source field at a given sensor divided by the field in the presence of the SIS. We observed shielding factors for sensors above the SIS brim varying from 50 to 200. As expected, sensors nearest the edge of the SIS experienced the poorest shielding varying between a factor of 15 and 30 depending on exact location. These shielding factors are, by nature of superconductors, independent of frequency. Trends in experimentally measured shielding factors agreed well with model predictions, however discrepancies in absolute shielding factors of up to $50 \%$ were observed. These were attributed to the pervasive presence of magnetic materials in and around our laboratory that could not be readily modeled.

Phantom sources were activated using a constantcurrent supply sinusoidally driven at $77 \mathrm{~Hz}$. The typical signal-to-noise $(\mathrm{S} / \mathrm{N})$ for acquired SQUID sensor data (for the sensor with maximal signal) was $\sim 20$. Data were acquired for 10 seconds resulting in an equivalent of 770 epochs.

Sensor calibration results (obtained by inverting the complete set of phantom data for each individual sensor) showed that SQUID localization errors were typically less than $0.5 \mathrm{~mm}$ and $20 \mathrm{mRad}$, however a few sensors were mislocated by more than $1 \mathrm{~mm}$. These mislocations were directly attributed to post-fabrication modifications. The impact of spatially calibrating the SQUID sensor array is evident in the phantom source localization results (Figures 2 and 3). Prior to calibrating sensor locations, the mean source localization error was $\sim 1 \mathrm{~mm}$ with the

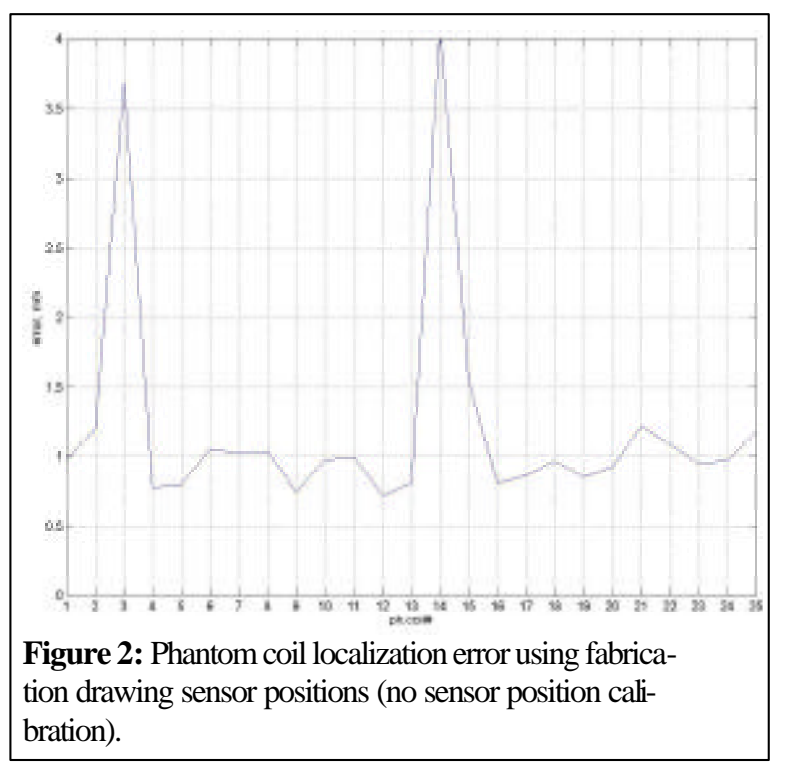




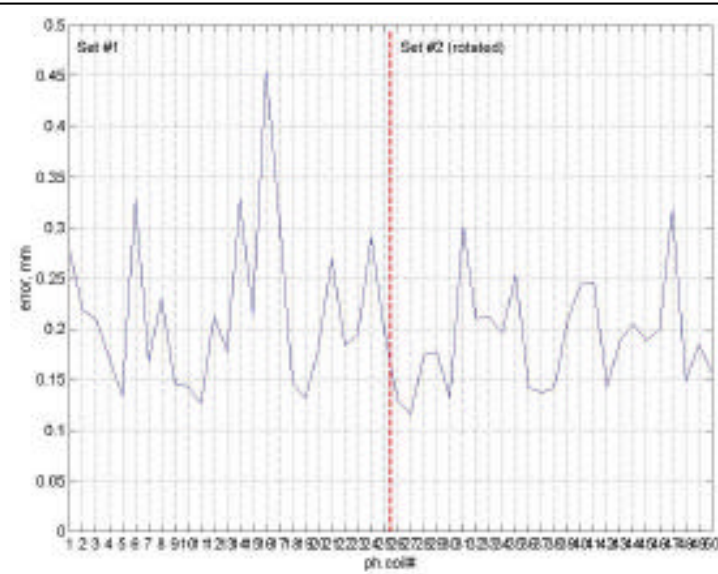

Figure 3: Extraordinary phantom coil localization precision using 'calibrated' sensor positions.

maximum error $\sim 4 \mathrm{~mm}$ (Figure 2). After calibrating the sensor locations, the means source localization error was $<0.2 \mathrm{~mm}$ with the maximum error of $0.45 \mathrm{~mm}$ (Figure 3 ).

Inverse source localization was performed to locate each of the 50 phantom coils and resulted in a mean error of less than 0.2 millimeters. This localization accuracy is more than a factor of five (5) better than any previously published result and an order of magnitude better than published figures for commercial whole-head MEG systems. Further, the accuracy of source localization for our system was independent of source orientation, unlike other systems where source orientation dramatically impacts source localization accuracy. Although the reason for this observation has not been determined, we speculate it may result form our choice of phantom source.

\section{Conclusion}

In conclusion, results obtained for the unique Los Alamos whole-head MEG system are very encouraging. Fields from external sources (noise) were typically reduced by approximately two orders of magnitude across all frequencies. This reduction was less for sensors near the edge of the SIS helmet, as expected, and depended on the details of the noise source.

We also report that the SIS-MEG system has an fffective "instrumental" source localization accuracy of better than $0.5 \mathrm{~mm}$ for localized, well characterized sources. This source localization accuracy was observed throughout the volume inside the SIS helmet, including regions near the periphery of the sensor array.

The ultimate goal of the SIS-MEG system is to precisely localize sources within a human brain. A detailed understanding of how various measurement and modeling errors impact source localization must be obtained in order to accurately compare various results reported in the literature. More importantly, this understanding is crucial to relating how localization results for simple phantoms (such as the one used here) compare with complex 'realworld' sources such as the human brain.

\section{References}

[1] Hämäläinen, M., Hari, R., Ilmoniemi, R. J., Knuutila, J., and Lounasmaa, O. V., Rev. Mod. Physics 65, $413-497$ (1993)

[2] Kraus, Jr., R.H. Flynn, E.R., Overton, W., Espy, M.A., J.S. George, Matlashov, A., Peters, M.A. and Ruminer, P., Recent Advances in Biomagnetism, T. Yoshimoto, et al. Ed., p. 5 (1999); 11th Intl. Conf. Biomagnetism, Sendai, Japan, August 1998.

[3] Volegov, P., Kraus, Jr., R.H., Maharajh, K., Matlachov, A., Espy, M., and E.R.Flynn, these proceedings .

\section{Acknowledgements}

We gratefully acknowledge the support of the US National $\mathbf{h}$ stitutes of Health (NINDS), US Department of Energy (OBER) and the National Foundation for Functional Brain Imaging. 